\title{
Fatores associados à atividade física na deficiência visual
}

Factors associated with physical activity in visual impairment

Roger Lima Scherer

Universidade Federal de Santa Catarina, Brasil

rogerlscherer@gmail.com

Fábio Colussi Karasiak

Universidade Federal de Santa Catarina, Brasil

Adriano Ferreti Borgatto

Universidade Federal de Santa Catarina, Brasil

\section{Resumo:}

Objetivo: Verificar os fatores determinantes para a prática de atividade física regular no lazer em adultos com deficiência visual da Grande Florianópolis. Método: Foi realizada uma entrevista pré-estruturada e validada com 168 adultos com deficiência visual, membros da Associação Catarinense para Integração dos Cegos. Resultados: $44 \%$ estão na faixa recomendável para o IMC e $30 \%$ são considerados ativos atualmente, diferentemente destes indivíduos na infância, quando $63 \%$ eram considerados ativos. Nas análises de razão de chances, encontrou-se que aqueles que apresentavam comportamento ativo na infância possuem menores chances de serem ativos na vida adulta e aqueles que possuem ensino superior completo ou pós-graduação e foram moderadamente ativos na infância possuem mais chances de serem ativos do que os demais adultos com deficiência visual. Conclusão: Estes resultados mostram o aumento significativo de sedentarismo, sobrepeso e obesidade neste segmento populacional, aumentando ainda mais as chances de desenvolverem doenças crônicas não transmissíveis.

Palavras-Chave: Exercício, Comportamento, Cego, Baixa Visão.

\section{Abstract:}

The aim is to verify the determining factors for the practice of regular physical activity in leisure in adults with visual impairment in Greater Florianópolis. A pre-structured and validated interview was applied in 168 visually impaired adults, members of one Association for Integration of the Blind. The results show that $44 \%$ are in the range recommended for BMI and $30 \%$ considered active, unlike these individuals in childhood, when $63 \%$ of them were considered active. In the analysis of odds ratios, it was found that adults with active behavior in childhood have a lower chance of being active in adult life, and those who have completed full or post-graduate education and were moderately active in childhood are more likely to be active than other adults with visual impairment. These results show a significant increase in sedentary lifestyle, overweight and obesity in this population segment, increasing the chances of developing chronic noncommunicable diseases.

KEYWORDs: Exercise, Behavior, Blind, Low Vision.

\section{INTRODUÇÃO}

A atividade física regular atualmente é muito discutida na literatura com relação a sua importância em um estilo de vida saudável, que implica em uma melhor qualidade de vida do indivíduo. Segundo Nahas (2017), as pessoas com deficiência (motora, intelectual ou sensorial) tendem a ser menos ativas fisicamente, entretanto este grupo populacional possui as mesmas necessidades na prevenção de doenças e no incremento da qualidade de vida que a população em geral. $\mathrm{O}$ autor ainda menciona que os sintomas de ansiedade e depressão, podem ser reduzidos com o incremento de atividades físicas regulares, além disso, esta atividade irá promover socialização e aumento da percepção de bem-estar geral.

Segundo Scherer (2012), aquelas pessoas com algum tipo de deficiência que não possuem estímulos ou oportunidades para uma prática contínua de atividade física regular são mais propensas a tornarem-se sedentárias, decorrentes de maiores limitações, falta de acessibilidade, além do próprio preconceito com 
relação a suas possibilidades. (Carroll et al., 2014) afirmam que quase metade de todos os adultos com deficiência relataram serem inativos e que estes possuem aproximadamente $50 \%$ mais chance de relatarem o desenvolvimento de doenças crônicas associadas a inatividade, tais como doenças cardiovasculares, obesidades, entre outros. Os autores ainda afirmam que entre as pessoas com deficiência, aquelas com deficiência visual tendem a estarem entre os menos ativos fisicamente.

A deficiência visual é uma limitação sensorial que pode levar a pessoa a ter comprometimento em seu desenvolvimento motor, afetivo, social e cognitivo (Cidade \& Freitas, 2002). Langelaan et al. (2007), ao estudarem o impacto da deficiência visual na qualidade de vida de adultos, constataram que adultos com deficiência visual, quando comparados a adultos sem deficiência, apresentam um impacto negativo substancial sobre a qualidade de vida.

O baixo nível de atividade física regular em adultos com deficiência visual é um fato bem documentado na literatura (Paul D. Loprinzi \& Joyner, 2016; Paul D. Loprinzi, Pariser, \& Ramulu, 2014; Paul D. Loprinzi, Smit, Lin, Gilham, \& Ramulu, 2013; Paul D. Loprinzi, Zebardast, \& Ramulu, 2015; P.D. Loprinzi, Brodowicz, Sengupta, Solomon, \& Ramulu, 2014). Pessoas com deficiência visual, quando não estimuladas e auxiliadas para uma prática regular de atividade física, irão encontrar mais dificuldades em sair de suas casas, por causa da sua limitação visual, o que favorece um estilo de vida sedentário e aumenta os riscos de doenças ligadas ao sedentarismo (Scherer, 2012). Tais limitações podem contribuir para o baixo nível de atividade física e consequente aumento do risco de mortalidade por todas as causas, uma vez que a deficiência visual poderá acarretar em um maior medo de quedas, além do isolamento social e as limitadas oportunidades de lazer (Holbrook, Kang, \& Morgan, 2013).

Sendo assim, este trabalho tem como objetivo verificar os fatores determinantes para a prática de atividade física regular no lazer em adultos com deficiência visual da Grande Florianópolis.

\section{Método}

Este estudo transversal foi realizado na Grande Florianópolis - SC entre agosto e outubro de 2011. A população para esta pesquisa foi constituída por pessoas com deficiência visual, residentes na região da Grande Florianópolis (compreende os municípios de: Florianópolis, São José, Palhoça, Santo Amaro da Imperatriz, São Bonifácio, Anitápolis, Rancho Queimado, Águas Mornas, São Pedro de Alcântara, Angelina, Antonio Carlos, Biguaçu e Governador Celso Ramos). A amostra do estudo foi composta por pessoas com deficiência visual, com idade entre 18 e 59 anos completos, membros da Associação Catarinense para Integração dos Cegos (ACIC).

A amostra foi determinada por conveniência, objetivando-se fazer o levantamento com todos os associados da entidade estudada. Na entidade, há 776 associados cadastrados, sendo elegíveis para o estudo apenas 321 associados nessa faixa etária ( 18 a 59 anos). A amostra final foi composta por 168 adultos, considerando que 11 se recusaram a participar, 87 não tinha telefone nem endereço atualizados nos prontuários da associação e não conseguimos contato com aproximadamente 55 indivíduos para realizar a coleta. As informações coletadas foram baseadas em um roteiro de entrevista pré-estruturado e validado.

As informações foram armazenadas no programa Excel 2013, tabuladas no software SPSS, versão 22.0 for Windows, onde foram empregados os procedimentos estatísticos. Para todos os testes foi adotado um nível de significância de $5 \%(\mathrm{p} \leq 0,05)$ e intervalos de confiança de $95 \%$. Realizou-se procedimentos de estatística descritiva utilizando frequências absolutas e relativas, além dos valores mínimos e máximos. Na regressão logística binária ajustada foram utilizadas apenas as variáveis que apresentaram $p \leq 0,20$ na regressão logística binária bruta.

Para a estimativa da razão de chance de pessoas ativas e o IC $95 \%$ foi utilizada a regressão logística binária. Para esta análise a variável dependente, nível de prática regular de atividade física no lazer, foi classificada em inativa e ativa baseado nas recomendações da Organização Mundial da Saúde. Sendo que aqueles indivíduos 
inativos ou que cumprem pouco as recomendações foram classificados como inativos. Os indivíduos que cumprem parcialmente as recomendações ou que cumprem as recomendações foram classificados como ativos. As variáveis independentes foram: sexo, idade, resquício visual (baixa visão e cego), percentual do resquício, tempo que adquiriu a deficiência visual, estado civil, nível de escolaridade, tempo que dedica ao trabalho, IMC e o comportamento que possuía na infância (inativo ou ativo).

A pesquisa foi aprovada pelo Comitê de Ética em Pesquisa com Seres Humanos da Universidade Federal de Santa Catarina, sob o no 1930/2011 e cumpri os princípios éticos contidos na Declaração de Helsinki. Todos os participantes assinaram o Termo de Consentimento Livre e Esclarecido, constando todos os procedimentos que seriam realizados e as formas de contato para possíveis dúvidas e esclarecimentos.

\section{Resultados}

Dentre os 168 adultos com deficiência visual da Grande Florianópolis desta amostra, 51,8\% são homens, sendo que aproximadamente $40 \%$ estão entre 31 e 45 anos. Quando estratificado por percentual remanescente da visão, a predominância é de adultos cegos (47\%), seguidos por adultos que possuem entre 6 e $15 \%$ de visão (20,2\%). Cerca de 63\% dos adultos desenvolveram à deficiência visual até os 3 anos de idade, ou seja, de forma congênita. Aproximadamente $40 \%$ dos adultos cegos e $50 \%$ dos adultos com baixa visão possuem no mínimo ensino médio completo, totalizando $45 \%$ da amostra, aproximadamente.

A maior parte dos indivíduos residem com seus familiares $(81,5 \%)$ e cerca de $56 \%$ trabalham, sendo que os adultos cegos trabalham mais que aqueles com baixa visão (62\%; 51,7\% respectivamente), em que 33,3\% dedicam até 30 horas semanais para o trabalho e os cegos são os que mais dedicam horas para as atividades laborais. Quando analisado o índice de massa corporal, apenas 44\% da amostra está na faixa recomendável para este indicador relacionado a saúde. Aproximadamente $70 \%$ são considerados inativos, comportamento diferente destes indivíduos na infância, quando os mesmos eram considerados ativos (aproximadamente 63\%) conforme apresentado na Tabela 1. 
TABELA 1

Descrição da amostra em relação às características sócio demográficas, visuais e comportamentais dos adultos com deficiência visual da Grande Florianópolis, Santa Catarina, 2011 ( $\mathrm{n}=168)$

\begin{tabular}{|c|c|c|c|c|c|c|}
\hline & \multicolumn{2}{|c|}{ Total } & \multicolumn{2}{|c|}{ Cego } & \multicolumn{2}{|c|}{ Baixa Visão } \\
\hline & $\mathbf{n}$ & $\%$ & $\mathbf{n}$ & $\%$ & $\mathbf{N}$ & $\%$ \\
\hline \multicolumn{7}{|l|}{ Sexo } \\
\hline Masculino & 87 & 51,8 & 47 & 59,5 & 40 & 44,9 \\
\hline Feminino & 81 & 48,2 & 32 & 40,5 & 49 & 55,1 \\
\hline \multicolumn{7}{|l|}{ Idade (anos) } \\
\hline 18 a 30 & 58 & 34,5 & 23 & 29,1 & 35 & 39,3 \\
\hline 31 a 45 & 66 & 39,3 & 30 & 38,0 & 36 & 40,4 \\
\hline 46 a 59 & 44 & 26,2 & 26 & 32,9 & 18 & 20,2 \\
\hline \multicolumn{7}{|l|}{ Percentual Remanescente } \\
\hline Cego & 79 & 47,0 & 79 & 100,0 & 0 & 0,00 \\
\hline Até $5 \%$ de visão & 30 & 17,9 & $Q$ & 00,0 & 30 & 33,7 \\
\hline 6 a $15 \%$ de visão & 34 & 20,2 & 0 & 00,0 & 34 & 38,2 \\
\hline Acima de $16 \%$ de visão & 25 & 14,9 & Q & 00,0 & 25 & 28,1 \\
\hline \multicolumn{7}{|l|}{ Período da Incidência } \\
\hline Congênito (até os 3 anos de idade) & 106 & 63,1 & 49 & 62,0 & 57 & 64,0 \\
\hline Adquirido & 62 & 36,9 & 30 & 38,0 & 32 & 36,0 \\
\hline \multicolumn{7}{|l|}{ Situação Conjugal } \\
\hline Sem companheiro & 91 & 54,2 & 54 & 60,7 & 91 & 54,2 \\
\hline Com companheiro & 77 & 45,8 & 35 & 39,3 & 77 & 45,8 \\
\hline \multicolumn{7}{|l|}{ Nível de Escolaridade } \\
\hline Analfabeto / Fundamental Incompleto & 38 & 22,6 & 19 & 24,1 & 19 & 21,3 \\
\hline Fundamental Completo / Médio Incompleto & 53 & 31,5 & 29 & 36,7 & 24 & 27,0 \\
\hline Médio Completo / Superior Incompleto & 55 & 32,7 & 19 & 24,1 & 36 & 40,4 \\
\hline Superior Completo / Pós Graduação & 22 & 13,1 & 12 & 15,2 & 10 & 11,2 \\
\hline \multicolumn{7}{|l|}{ Com que mora } \\
\hline Sozinho & 16 & 9,5 & 4 & 5,1 & 12 & 13,5 \\
\hline Familiares & 137 & 81,5 & 65 & 82,3 & 72 & 80,9 \\
\hline Outras Pessoas & 15 & 8,9 & 10 & 12,7 & 5 & 5,6 \\
\hline \multicolumn{7}{|l|}{ Trabalha } \\
\hline Sim & 95 & 56,5 & 49 & 62,0 & 46 & 51,7 \\
\hline Não & 73 & 43,5 & 30 & 38,0 & 43 & 48,3 \\
\hline \multicolumn{7}{|l|}{ Dedicação para trabalho } \\
\hline Não Trabalha & 73 & 43,5 & 30 & 38,0 & 43 & 48,3 \\
\hline Até 30 horas & 56 & 33,3 & 25 & 31,6 & 31 & 34,8 \\
\hline Acima de 31 horas & 39 & 23,2 & 24 & 30,4 & 15 & 16,9 \\
\hline \multicolumn{7}{|l|}{ IMC } \\
\hline Faixa Recomendável & 74 & 44,0 & 30 & 38,0 & 44 & 49,4 \\
\hline Sobrepeso & 46 & 27,4 & 26 & 32,9 & 20 & 22,5 \\
\hline Obesidade & 48 & 28,6 & 23 & 29,1 & 25 & 28,1 \\
\hline \multicolumn{7}{|l|}{ Comportamento na Infância } \\
\hline Inativo & 11 & 6,5 & 4 & 5,1 & 7 & 7,9 \\
\hline Pouco Ativo & 18 & 10,7 & 10 & 12,7 & 8 & 9,0 \\
\hline Moderadamente Ativo & 32 & 19,0 & 16 & 20,3 & 16 & 18,0 \\
\hline Ativo & 107 & 63,7 & 49 & 62,0 & 58 & 65,2 \\
\hline \multicolumn{7}{|l|}{ Atividade Física Regular } \\
\hline Inativo & 118 & 70,2 & 58 & 73,4 & 60 & 67,4 \\
\hline Ativo & 50 & 29,8 & 21 & 26,6 & 29 & 32,6 \\
\hline
\end{tabular}

$\mathrm{Na}$ tabela 2 estão apresentadas as razões de chance bruta e ajustada para atividade física regular dos adultos com deficiência visual com as variáveis sócio demográficas, visuais e comportamentais. 
TABELA 2

Razão de chances para praticar atividade física regular em adultos com deficiência visual segundo variáveis sócio demográficas, visuais e comportamentais. Grande Florianópolis, Santa Catarina, 2011 ( $\mathrm{n}=168)$

\begin{tabular}{|c|c|c|c|c|}
\hline & \multicolumn{2}{|l|}{ BRUTA } & \multicolumn{2}{|c|}{ AJUSTADA } \\
\hline & $\mathrm{RC}(\mathrm{IC} 95 \%)$ & p-valor & RC (IC95\%) & p-valor \\
\hline Sexo & & 0,295 & $\ldots$ & -- \\
\hline Masculino & $1,43(0,73-2,78)$ & & $\ldots$ & $\ldots$ \\
\hline Feminino & 1,00 & & $\ldots$ & $-\ldots$ \\
\hline Idade & & 0,992 & $\ldots$ & $\ldots$ \\
\hline 18 a 30 anos & $0,99(0,42-2,33)$ & & 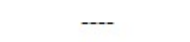 &.-- \\
\hline 31 a 45 anos & $1,04(0,45-2,38)$ & & $\ldots$ & $\ldots$ \\
\hline 46 a 59 anos & 1,00 & & $\ldots$ & $\ldots$ \\
\hline Resquício Visual & & 0,396 & $\ldots$ & $\ldots$ \\
\hline Cego & $0,75(0,72-2,74)$ & & --.- & -..- \\
\hline Baixa Visão & 1,00 & & $\ldots$ & $\ldots$ \\
\hline Percentual Remanescente & & 0,866 & $\ldots$ & $\ldots$ \\
\hline Cego & $0,77(0,29-2,04)$ & & $\cdots$ & $\cdots$ \\
\hline Até $5 \%$ & $1,06(0,34-3,28)$ & & -..- & $\ldots$ \\
\hline 6 a $15 \%$ & $1,01(0,33-3,07)$ & & $\ldots$ & $\ldots$ \\
\hline Acima de $16 \%$ & 1,00 & & --- & $\ldots$ \\
\hline Período da Incidência & & 0,374 & $\cdots$ & $\ldots$ \\
\hline Congênito & $0,73(0,37-1,45)$ & & 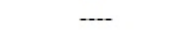 & $\ldots$ \\
\hline Adquirido & 1,00 & & $\ldots$ & $\ldots$ \\
\hline Situação Conjugal & & 0,297 & --- & --. \\
\hline Sem companheiro & $0,70(0,36-1,36)$ & & -..- & --.- \\
\hline Com companheiro & 1,00 & & $\ldots$ & $\ldots$ \\
\hline Nível de Escolaridade & & 0,181 & & 0,483 \\
\hline Analfabeto / Fundamental Incompleto & $0,27(0,08-0,87)$ & & $0,38(0,11-1,33)$ & \\
\hline Fundamental Completo / Médio Incompleto & $0,57(0,20-1,57)$ & & $0,71(0,24-2,12)$ & \\
\hline Médio Completo / Superior Incompleto & $0,49(0,18-1,37)$ & & $0,63(0,21-1,86)$ & \\
\hline Superior Completo / Pós Graduação & 1,00 & & 1,00 & \\
\hline Com que mora & & 0,377 & $-\ldots$ & $\cdots$ \\
\hline Sozinho & $2,95(0,47-18,34)$ & & $\ldots$ & $\ldots$ \\
\hline Familiares & $2,97(0,64-13,76)$ & & $\ldots$ & $\ldots$ \\
\hline Outras Pessoas & 1,00 & & $\ldots$ & $\ldots$ \\
\hline Trabalha & & 0,557 &.-- & -- \\
\hline Sim & $1,22(0,62-2,39)$ & & --- & --- \\
\hline Não & 1,00 & & $\ldots$ & -- \\
\hline Dedicação para trabalho & & 0,479 & $\cdots$ & $\cdots$ \\
\hline Não Trabalha & $0,89(0,37-2,14)$ & & -- & -- \\
\hline Até 30 horas & $1,41(0,58-3,43)$ & & $\ldots$ & $\ldots$ \\
\hline Acima de 31 horas & 1,00 & & --. & $\ldots$ \\
\hline IMC & & 0,516 & --.- & -.- \\
\hline Sobrepeso & $0,61(0,27-1,41)$ & & $\ldots$ & $\ldots$ \\
\hline Obesidade & $0,81(0,37-1,78)$ & & --- & $-\cdots$ \\
\hline Faixa Recomendável & 1,00 & & $\ldots$ & $\ldots$ \\
\hline Comportamento na Infância & & 0,006 & & 0,019 \\
\hline Inativo & $1,30(0,32-5,27)$ & & $1,48(0,35-6,21)$ & \\
\hline Pouco Ativo & $1,33(0,43-4,10)$ & & $1,42(0,45-4,47)$ & \\
\hline Moderadamente Ativo & $4,44(1,93-10,23)$ & & $3,94(1,68-9,22)$ & \\
\hline Ativo & 1,00 & & 1,00 & \\
\hline
\end{tabular}


Não houve diferenças significativas em nenhuma variável exceto no comportamento relacionado a atividade física na infância, que apresentou associação com o nível de atividade física regular no lazer $(p=0,006)$ sendo que aqueles que eram moderadamente ativos na infância apresentaram $344 \%$ a mais de chance de serem ativos que aqueles que eram ativos na infância.

$\mathrm{Na}$ análise ajustada foi incluída apenas as variáveis de nível de escolaridade e comportamento na infância. Sendo que aqueles que possuem ensino superior completo ou pós-graduação possuem mais chances de serem ativos que os demais. O comportamento na infância apresentou associação com o nível de atividade física regular no lazer, sendo que aqueles que eram ativos na infância apresentaram menor chance de serem ativos quando adultos que os demais indivíduos. Desta forma, após controlar alguns possíveis valores de confundimento, adultos com ensino superior completo ou pós-graduação que foram moderadamente ativos na infância possuem mais chance de serem ativos que os demais adultos com deficiência visual.

\section{Discussão}

A idade média dos indivíduos nesta pesquisa foi de 37,15 anos $( \pm 11,21)$, média abaixo dos estudos de Becker \& Montilha (2015) e Bittencourt, Montilha, Gasparetto, Temporini, \& Carvalho (2011), com média de 46,7 e 41 anos, respectivamente. Já com relação ao resquício visual, esta pesquisa encontrou cerca de 53\% de adultos com baixa visão, valores abaixo da pesquisa realizada por Becker \& Montilha (2015) com cerca de $74 \%$ de pessoas com baixa visão e 65,2\% encontrados por Bittencourt, Montilha, Gasparetto, Temporini, \& Carvalho (2011). Aproximadamente 63\% dos adultos desta pesquisa são considerados congênitos com relação a incidência da deficiência visual. Segundo Mosquera (2000) as pessoas com deficiência visual são consideradas congênitas caso a adquiram até os três anos de idade, pois antes desta idade ela não grava imagens, ou forma conceitos do ambiente ou lugar.

Com relação ao percentual remanescente de visão, $54 \%$ deste grupo apresenta a baixa visão, tendo aproximadamente $20 \%$ destes entre 6 e $15 \%$ de visão, o que contribui de forma significativa na autonomia e independência da pessoa.

A deficiência visual por si só não é a responsável por comprometer o desenvolvimento pleno do indivíduo. Haegele, Hodge, \& Kozub (2017) afirmam que não há uma identificação concreta e análise de fatores que influenciam o envolvimento em atividades físicas e comportamentos sedentários entre os adultos com deficiência visual. Contudo, a falta de oportunidades que esta pessoa passa a ter em virtude da sua limitação visual pode comprometer o desenvolvimento pleno do indivíduo. Assim, devido à diminuição da independência, a deficiência visual pode ser um indicativo para um estilo de vida inativo (Ray, Horvat, Williams \& Blasch, 2007).

Quando analisado o índice de massa corporal, apenas $44 \%$ da amostra está na faixa recomendável para este indicador relacionado a saúde. E aproximadamente $30 \%$ são considerados ativos. Comportamento diferente destes indivíduos na infância, quando aproximadamente $63 \%$ dos mesmos eram considerados ativos. Ray, Horvat, Williams, \& Blasch (2007) afirmam que a redução do funcionamento físico, juntamente com o aumento do IMC poderá acarretar na diminuição da mobilidade assim como aumentar o risco de doenças crônicas entre as pessoas com deficiência visual. Para o National Institutes of Health (1998), a obesidade e o sobrepeso aumentam de forma substancial o risco de morbidades, tais como: dislipidemias, hipertensão, diabetes tipo 2, problemas respiratórios, doenças cardiovasculares, alguns tipos de câncer, entre outros. $\mathrm{O}$ aumento do peso também está associado a mortalidade por diversas causas, sendo que a relação entre o peso corporal e o risco de doença é um fator contínuo, pois a medida que o peso aumenta o risco de doenças também aumenta. Capella-McDonnall (2007), em seu estudo com dados de instituições como Center for Disease Control and Prevention (CDC) e National Health Interview Survey, derivados de pessoas com deficiência, encontraram que estas apresentam maiores taxas de sobrepeso e obesidade que pessoas sem 
deficiência. As pessoas com deficiência visual apresentam 1,5 vez mais chance de serem obesas que a população em geral. Na presente pesquisa, observou-se que a cada 10 adultos, seis estão fora da faixa recomendável, sendo três obesos.

Em pesquisa realizada em 2011, através do programa de Vigilância de fatores de risco e proteção para doenças crônicas por inquérito telefônico com a população de maneira geral acima dos 18 anos, Monteiro et al. (2011) encontraram que $41,4 \%$ dos adultos praticam atividade física com o volume recomendado pela World Health Organization (2010).

Alguns autores associam o nível de atividade física na fase adulta com o índice de comportamento sedentário observado na infância e adolescência (Guedes \& Guedes, 2006). Bauman (2004) reforça esta constatação, afirmando que acompanhamentos longitudinais sugerem que adolescentes menos ativos fisicamente apresentam maior predisposição para se tornarem adultos sedentários. Entretanto, Seron, Interdonato, Junior, Corrêa, \& Greguol (2012) afirmam que há uma carência de estudos relacionando a prática habitual de atividade física envolvendo crianças e adolescentes e associando com o nível de atividade física durante a fase adulta.

O principal achado deste estudo foi encontrar dados diferentes aos da população sem deficiência visual, com relação ao nível de atividade física na infância/adolescência e na fase adulta. Entre os adultos que apresentavam comportamento ativo ou moderadamente ativo na infância (63\% e 19\%, respectivamente), apenas 30\% apresentaram estes mesmos comportamentos quando adultos.

$\mathrm{Na}$ tabela 2 do presente estudo, foram apresentadas as razões de chance bruta e ajustada para atividade física regular com variáveis sócio demográficas, visuais e comportamentais. $\mathrm{Na}$ análise bruta e na ajustada, não houve diferenças significativas em nenhuma variável, exceto no comportamento relacionado a atividade física na infância. Haegele et al. (2017) complementam que, embora as pesquisas identifiquem uma série de variáveis sócio demográficas que demonstram impactar a atividade física para pessoas sem deficiência tais como nível de renda e índice de massa corporal -, estas variáveis podem não prever o nível de atividade física entre as pessoas com deficiência visual. Assim como a idade em que ocorreu a incidência da deficiência visual poderá também não afetar o nível de atividade física do indivíduo (Marmeleira, Laranjo, Marques, \& Pereira, 2014). Haegele, Zhu, Lee, \& Lieberman (2016) constataram que o percentual de visão remanescente também não está associado com o nível de atividade física. Sendo que em sua pesquisa o mesmo encontrou apenas associação com sexo, já o nível de escolaridade do grupo pesquisado ( $\mathrm{n}=176$ adultos) quase apresentou associação com o nível de atividade física $(\mathrm{p}=0,06)$.

Quando o nível de atividade física foi ajustado para comportamento na infância e nível de escolaridade. Desta forma, após controlar possíveis valores que confundam a análise, constatou que aqueles que possuem ensino superior completo ou pós-graduação e que foram moderadamente ativos na infância possuem mais chances de serem ativos que os demais adultos com deficiência visual, corroborando com os estudos de Guedes \& Guedes (2006) e Bauman (2004) - sobre a importância de ser ativo na infância. Entretanto, aqueles que eram ativos na infância, possuem menos chances de serem ativos durante a fase adulta, inclusive quando comparados àqueles que eram inativos durante a infância.

Isso pode ser decorrente das barreiras impostas pela sociedade e seu próprio preconceito com relação a suas possibilidades e capacidades. Estes são fatores determinantes para uma vida mais ativa por parte das pessoas com deficiência visual. Segundo Kodish, Kulinna, Martin, Pangrazi, \& Darst (2006) muitas vezes a prática de atividade física na infância e adolescência pode ser comprometida pela falta de oportunidades na participação em programas organizados de atividade física, inclusive na própria educação física escolar. $\mathrm{O}$ aumento das oportunidades, as modificações do meio e as melhorias das atividades oferecidas facilitam e oportunizam uma vida mais ativa às pessoas com deficiência (Marques, 2005). Paul D. Loprinzi \& Joyner (2016) afirmam que são necessários esforços para identificar estratégias efetivas para aumentar o nível de atividade física entre os adultos com deficiência visual. 
Outra hipótese que pode ser considerada é com relação àqueles indivíduos que eram ativos na infância terem adquirido a deficiência visual durante a fase adulta acarretando em uma diminuição significativa do seu nível de atividade física. Além disso, podemos levantar também aspectos como os compromissos com trabalho, estudo e reabilitação. Uma combinação destes aspectos com a limitação visual e barreiras físicas e atitudinais pode provocar uma diminuição do nível de atividade física da infância na fase adulta. Entretanto aqueles que eram moderadamente ativos na infância podem ter mantido o nível de atividade física ou aumentado de forma discreta, visto que o desfecho nível de atividade física foi dicotomizado em inativo (inativo ou cumpre pouco as recomendações) e ativo (cumpre parcialmente as recomendações e cumpre as recomendações). Entretanto, como afirmam Furtado, Allums-Featherston, Lieberman, \& Gutierrez (2015), são poucos os estudos de intervenção em crianças com deficiência visual com relação a atividade física regular, sendo que os poucos conduzidos não possuem um bom delineamento. Ficando desta forma uma lacuna sobre a mudança dos níveis de atividade física na infância e sua relação direta no nível de atividade física no adulto.

\section{CoNClusão}

Podemos concluir que mais de $50 \%$ da amostra encontra-se fora da faixa recomendada com relação ao indicador relacionado a saúde (IMC). Sendo que o mesmo, quando relacionado com um número preocupante de $70 \%$ de adultos com deficiência visual inativos, pode acarretar em futuras doenças crônicas não transmissíveis.

O nível de atividade física apresentou associação durante as análises bruta e ajustada apenas com o comportamento durante a infância com relação a atividade física. Apesar destes adultos durante sua infância serem em sua grande maioria ativos (63\%), aqueles que eram moderadamente ativos na infância (19\%) possuem mais chances de serem ativos na fase adulta do que os demais. Estes números são preocupantes, principalmente no que concerne ao aumento significativo de indivíduos com sobrepeso, obesidade e inativos dentro deste segmento populacional, aumentando assim as chances de desenvolverem doenças crônicas não transmissíveis na fase adulta. Diante disto, recomenda-se outros estudos para investigar esta temática, bem como para encontrar melhores estratégias e subsídios para aumentar o nível de atividade física desta população.

Apesar das conclusões do presente estudo, também devemos levar em consideração algumas limitações, pois o mesmo foi conduzido com uma amostra restrita (168 indivíduos) e proveniente de uma mesma instituição, sendo interessante aumentar a amostra e procurar grupos de diferentes localidades e instituições para conclusões mais precisas.

\section{REFERÊNCIAS}

Bauman, A. E. (2004). Updating the evidence that physical activity is good for health: an epidemiological review 2000-2003. Journal of Science and Medicine in Sport, 7(1 Suppl), 6-19.

Becker, P., \& Montilha, R. de C. I. (2015). Ocupational performance and quality of life: interrelationships in daily life of visual impaired individuals. Revista Brasileira de Oftalmologia, 74(6), 372-377. https:// doi.org/10.5935/0034-7280.20150078

Bittencourt, Z. Z. L. de C., Montilha, R. de C. L., Gasparetto, M. E. R. F., Temporini, E. R., \& Carvalho, K. M. M. de. (2011). Diabetic retinopathy and visual disabilities among patients in a rehabilitation program. Revista Brasileira de Oftalmologia, 70(6), 342-348.

Capella-McDonnall, M. (2007). The need for health promotion for adults who are visually impaired.Journal of Visual Impairment \& Blindness, 101(3), 133. 
Carroll, D. D., Courtney-Long, E. A., Stevens, A. C., Sloan, M. L., Lullo, C., Visser, S. N., ... Dorn, J. M. (2014). Vital signs: disability and physical activity -- United States, 2009-2012. Morbidity and Mortality Weekly Report, 63(18), 407-413.

Cidade, R. E. A., \& Freitas, P. S. de. (2002). Introdução à Educação Física e ao Desporto para Pessoas Portadoras de Deficiência (lo ed). Curitiba: UFPR - Editora Universidade. Recuperado de http://www.tblivraria.com.br/livro-introducao-a-educacao-fisica-e-ao-desporto-para-pessoasportadoras-de-deficiencia-9788573350937,am2651.html

Furtado, O. L. P. C., Allums-Featherston, K., Lieberman, L. J., \& Gutierrez, G. L. (2015). Physical Activity Interventions for Children and Youth with Visual Impairments. Adapted Physical Activity Quarterly, 32(2), 156-176. https://doi.org/10.1123/APAQ.2014-0164

Guedes, D. P., \& Guedes, J. E. R. P. (2006). Manual prático para avaliação em educação física. Barueri: Manole.

Haegele, J. A., Hodge, S. R., \& Kozub, F. M. (2017). Beliefs about physical activity and sedentary behaviors of adults with visual impairments. Disability and Health Journal, 10(4), 571-579. https://doi.org/10.1016/ j.dhjo.2017.03.008

Haegele, J. A., Zhu, X., Lee, J., \& Lieberman, L. J. (2016). Physical activity for adults with visual impairments: impact of socio-demographic factors. European Journal of Adapted Physical Activity, 9(1), 3-14. Recuperado de http:// eujapa.upol.cz/index.php/EUJAPA/article/view/182

Holbrook, E. A., Kang, M., \& Morgan, D. W. (2013). Acquiring a stable estimate of physical activity in adults with visual impairment. Adapted Physical Activity Quarterly, 30(1), 59-69.

Kodish, S., Kulinna, P. H., Martin, J., Pangrazi, R., \& Darst, P. (2006). Determinants of physical activity in an inclusive setting. Adapted Physical Activity Quarterly, 23(4), 390-409.

Langelaan, M., Boer, M. R. de, Nispen, R. M. A. van, Wouters, B., Moll, A. C., \& Rens, G. H. M. B. van. (2007). Impact of Visual Impairment on Quality of Life: A Comparison With Quality of Life in the General Population and With Other Chronic Conditions. Ophthalmic Epidemiology, 14(3), 119-126. https:// doi.org/10.1080/09286580601139212

Loprinzi, Paul D., \& Joyner, C. (2016). Accelerometer-determined physical activity and mortality in a national prospective cohort study: Considerations by visual acuity. Preventive Medicine, 87, 18-21. https:// doi.org/10.1016/j.ypmed.2016.02.005

Loprinzi, Paul D., Pariser, G., \& Ramulu, P. Y. (2014). Accelerometer-Assessed Sedentary and Physical Activity Behavior and Its Association with Vision among U.S. Adults with Diabetes. Journal of Physical Activity and Health, 11(6), 1156-1161. https://doi.org/10.1123/jpah.2012-0354

Loprinzi, Paul D., Smit, E., Lin, F. R., Gilham, B., \& Ramulu, P. Y. (2013). Accelerometer-Assessed Physical Activity and Objectively Determined Dual Sensory Impairment in US Adults. Mayo Clinic Proceedings, 88(7), 690-696. https://doi.org/10.1016/j.mayocp.2013.04.008

Loprinzi, Paul D., Zebardast, N., \& Ramulu, P. Y. (2015). Cardiorespiratory fitness and vision loss among young and middle-age US adults. American Journal of Health Promotion, 29(4), 226-229.

Loprinzi, P.D., Brodowicz, G. R., Sengupta, S., Solomon, S. D., \& Ramulu, P. Y. (2014). Accelerometer-assessed physical activity and diabetic retinopathy in the United States.JAMA Ophthalmology, 132(8), 1017-1019.

Marmeleira, J., Laranjo, L., Marques, O., \& Pereira, C. (2014). Physical Activity Patterns in Adults Who Are Blind as Assessed by Accelerometry. Adapted Physical Activity Quarterly, 31(3), 283-296. https://doi.org/10.1123/ apaq.2013-0039

Marques, A. C. (2005). O papel da atividade física na saúde e qualidade de vida de pessoas deficientes. In Revista Brasileira de Atividade Física \& Saúde, Anais... Florianópolis.

Monteiro, C. A., Claro, R. M., Malta, D. C., Martins, A. P. B., Iser, B. P. M., Sá, N. N. B., ... Bernal, R. T. I. (2011). Vigilância de fatores de risco e proteção para doenças crônicas por inquérito telefônico: Estimativas sobre frequência e distribuição sociodemográfica de fatores de risco e proteção para doenças crônicas nas capitais dos 26 Estados brasileiros e no Distrito Federal em 2011. Brasília: Ministério da Saúde, Secretaria de Vigilância em Saúde. 
Mosquera, C. (2000). Educação Física para Deficientes Visuais. Rio de Janeiro: SPRINT. Recuperado de https:// www.estantevirtual.com.br/b/carlos-mosquera/educacao-fisica-para-deficientes-visuais/3596895243

Nahas, M. V. (2017). Atividade Física, Saúde \& Qualidade de Vida: Conceitos e Sugestões para um Estilo de Vida Ativo (7o ed). Florianópolis: do Autor.

National Institutes of Health (1998). Clinical Guidelines on the Identification, Evaluation, and Treatment of Overweight and Obesity in Adults: The Evidence Report. National Heart, Lung, and Blood Institute.

Ray, C., Horvat, M., Williams, M., \& Blasch, B. (2007). Kinetic movement analysis in adults with vision loss. Adapted Physical Activity Quarterly: APAQ, 24(3), 209-217.

Scherer, R. L. (2012). Qualidade de Vida de Adultos com Deficiência Visual da Grande Florianópolis (Dissertação). Universidade Federal de Santa Catarina, Florianópolis.

Seron, B. B., Interdonato, G. C., Junior, L., Corrêa, C., \& Greguol, M. (2012). Habitual physical activity among adolescents with visual impairments. Revista Brasileira de Educação Física e Esporte, 26(2), 231-239. https:// doi.org/10.1590/S1807-55092012000200006

World Health Organization. (2010). Global recommendations on physical activity for health. Geneva: World Health Organization. Recuperado de http://www.ncbi.nlm.nih.gov/books/NBK305057/ 